\title{
ANTI-HERPESVIRUS ACTIVITY OF CARBOCYCLIC OXETANOCIN G IN VITRO
}

\author{
Yukihiro Nishiyama, Naohiko Yamamoto, Yoshinari Yamada, Tohru Daikoku, \\ YUH-ICHIRO ICHIKAWA ${ }^{\dagger}$ and KaTSUTOSHI TAKAHASHI ${ }^{\dagger}$ \\ Laboratory of Virology, Research Institute for Disease Mechanism and Control, \\ Nagoya University School of Medicine, \\ Nagoya 466, Japan \\ ${ }^{\dagger}$ Research Laboratories, Pharmaceutical Group, Nippon Kayaku Co., Ltd., \\ Tokyo 115, Japan
}

(Received for publication August 11, 1989)

\begin{abstract}
A series of new compounds, carbocyclic oxetanocins, have been synthesized and their anti-herpesvirus activity determined. Carbocyclic oxetanocin G (OXT-G) was most active against herpes simplex virus (HSV) and human cytomegalovirus (HCMV) among carbocyclic oxetanocins tested; the median effective concentrations $\left(\mathrm{EC}_{50}\right)$ for $\mathrm{HSV}-1,-2$, and $\mathrm{HCMV}$ were $0.23,0.04$ and $0.40 \mu \mathrm{g} / \mathrm{ml}$, respectively. The $\mathrm{EC}_{50}$ value of carbocyclic OXT-G against HSV-2 was significantly lower than those of acyclovir, ganciclovir (DHPG) and OXT-G, while the value for HCMV was comparable to those of DHPG and OXT-G. Carbocyclic OXT-G showed much higher activity against $\mathrm{TK}^{+}$HSV-2 than against a $\mathrm{TK}^{-}$mutant, suggesting that this compound is a good substrate for HSV-2-induced TK. The antiviral activity of the compound was only partially reversed even by the addition of 100-fold excess deoxyguanosine. The results suggest that the mode of action of carbocyclic OXT-G is different from that of OXT-G.
\end{abstract}

Acyclovir (ACV) is the most successful antiviral agent that has been licenced for the treatment of herpes simplex virus (HSV) infections, and has proved beyond all doubt that antiviral therapy with minimal toxicity is an achievable goal. But this agent is not so active against HSV type 2 (HSV-2) as against HSV type 1 (HSV-1 $)^{1,2)}$, and has shown little efficacy in the treatment of human cytomegalovirus (HCMV) infections ${ }^{1 \sim 4)}$. Ganciclovir (DHPG), a potent inhibitor against HCMV in vitro, is currently undergoing clinical trials for the treatment of HCMV infections in bone marrow transplant recipients and in patients with aquired immunodeficiency syndrome (AIDS) ${ }^{5 \sim 8)}$. Although ganciclovir has demonstrated significant antiviral activity against HCMV in these patients, the therapeutic efficacy is limited by its toxicity, principally ganciclovir-induced myelosuppression ${ }^{9 \sim 11)}$. Therefore it is necessary to keep on searching more selective and potent agents against these pathogens. Recently we have reported that oxetanocin G (OXT-G), a novel nucleoside having an oxetanocyl- $N$-glycoside linkage, has a potent and selective antiviral activity against HCMV in vitro ${ }^{12)}$. OXT-G was also highly effective against systemic HSV-2 and murine CMV infections in mice ${ }^{13)}$. As part of our antiviral chemotherapy program, we had an interest in carbocyclic analogs of oxetanocins. In the present study, we investigated the effect of these compounds against HSV and HCMV in vitro.

\section{Materials and Methods}

Chemicals

OXT-G were synthesized as described previously ${ }^{14)}$. NNU-018 and -019 were synthesized from $(+)-(1 \alpha, 3 \beta)-3-(t e r t$-butyldiphenylsilyloxymethyl)cyclobutane-1-yl methansulfonate with adenine or 
Fig. 1. Structures of carbocyclic oxetanocins.
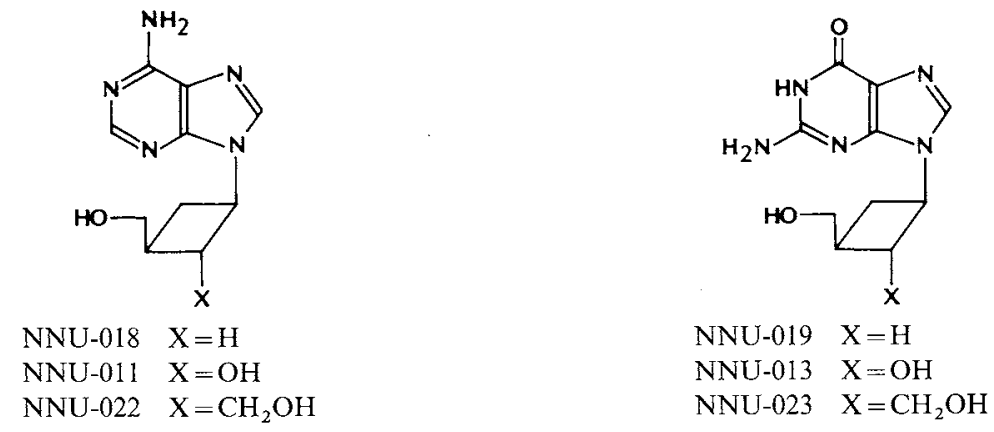

2-amino-6-(2-methoxyethoxy)purine, followed by deprotection. NNU-011 and -013 were synthesized from

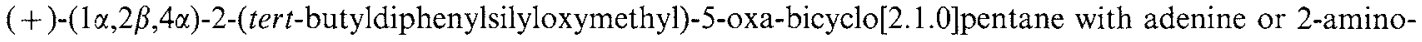
6-(2-methoxyethoxy)purine, followed by deprotection. Procedures for chemical synthesis of these four compounds will be described in detail elsewhere. NNU-022 and -023 were synthesized from (+)-(1S,2S,3S)-2,3-bis(tert-butyldiphenylsilyloxymethyl)cyclobutane-1-yl methansulfonate with adenine or 2-amino-6-(2-methoxyethoxy)purine, followed by deprotection as reported previously ${ }^{15)}$. Optical purities of NNU-022 and -023 were determined from NMR spectrum of bis- $(R)-\alpha$-methoxy- $\alpha$-trifluoromethylphenylacetate of an intermediate (-)-(2S,3S)-2,3-bis(hydroxymethyl)-1,1-bis(methylthio)cyclobutane, and were $>98 \%$ ee. All compounds were characterized by $400 \mathrm{MHz} \mathrm{NMR}$, UV, and high-resolution fast atom bombardment (HRFAB)-MS. Purities of these compounds were determined by HPLC, and were $>98 \%$. The chemical structures of carbocyclic oxetanocins used in this study are shown in Fig. 1. ACV and DHPG were provided by Burroughs Wellcome Co., Research Triangle Park, N.C., and Syntex Laboratories, Inc., Palo Alto, Calif., respectively.

\section{Cells and Viruses}

Vero cells, a line of African green monkey kidney cells, and human embryonic fibroblasts (HEF) were used in this study and were grown in EAGLE's minimum essential medium (MEM) supplemented with 7\% calf serum (CS) and 10\% fetal calf serum (FCS), respectively. HSV-1 strain HF, HSV-2 strain 186 (wild type $\left[\mathrm{TK}^{+}\right]$and thymidine kinase deficient $\left[\mathrm{TK}^{-}\right]$), and HCMV strain AD169 were propagated in HEF cell monolayers as described previously ${ }^{16,17)}$.

\section{Plaque Reduction Assays}

Confluent monolayers of Vero or HEF cells in plastic dishes (diameter, $35 \mathrm{~mm}$ ) were infected with 100 to 150 plaque forming units (PFU) of HSV or HCMV. After a 1-hour adsorption period at $37^{\circ} \mathrm{C}$, the cultures were overlaid with $2 \mathrm{ml}$ of $0.5 \%$ agarose in MEM containing $3 \% \mathrm{FCS}$ and various concentrations of drugs. The cultures infected with HSV or HCMV were fixed and stained at 1 or 2 and 9 or 10 days after infection, respectively. In the case of HCMV-infected cultures, the second agarose overlay containing appropriate concentrations of drugs was added 5 days after infection. Plaque numbers were counted by using a dissecting microscope at $\times 20$ magnification.

Yield Reduction Assays

Monolayers of Vero or HEF cells were infected with HSV-2 or HCMV at a multiplicity of about 10 PFU per cell and treated with various concentrations of drugs after a 1-hour virus adsorption period at $37^{\circ} \mathrm{C}$. HSV-2 and HCMV were harvested 24 hours and 5 days postinfection, respectively. After freeze-thawing followed by low speed centrifugation, the supernatant was assayed for virus infectivity.

\section{Results}

Antiviral Activities of Carbocyclic Oxetanocins

The antiviral activities of carbocyclic oxetanocins were measured by the plaque reduction assays and 
Table 1. Antiviral activities of carbocyclic oxetanocins.

\begin{tabular}{cccc}
\hline & \multicolumn{3}{c}{$\mathrm{EC}_{50}(\mu \mathrm{g} / \mathrm{ml})^{\mathrm{a}}$} \\
\cline { 2 - 4 } Compound & HSV-1 & HSV-2 & HCMV \\
\hline NNU-018 & $>20$ & $>20$ & $>20$ \\
NNU-011 & 3.4 & 4.2 & 0.45 \\
NNU-022 & 8.0 & 7.5 & 12 \\
NNU-019 & 8.0 & 2.0 & 2.6 \\
NNU-013 & 0.74 & 0.12 & 0.95 \\
NNU-023 & 0.23 & 0.04 & 0.40
\end{tabular}

a Results are the average of two or three different experiments. Each experiment was carried out in duplicate or triplicate.
Table 2. Inhibitory effects of NNU-023, OXT-G, ACV, and DHPG against wild-type and $\mathrm{TK}^{-} \mathrm{HSV}-2$.

\begin{tabular}{lcc}
\hline \multirow{2}{*}{ Compound } & \multicolumn{2}{c}{$\mathrm{EC}_{50}(\mu \mathrm{g} / \mathrm{ml})^{\mathrm{a}}$} \\
\cline { 2 - 3 } & $\mathrm{HSV}-2\left(\mathrm{TK}^{+}\right)$ & $\mathrm{HSV}-2\left(\mathrm{TK}^{-}\right)$ \\
\hline NNU-023 & 0.04 & 1.4 \\
OXT-G & 2.0 & 2.4 \\
ACV & 0.18 & 12 \\
DHPG & 0.30 & 64 \\
\hline
\end{tabular}

a Results are the averages of two experiments. Each experiment was carried out in duplicate or triplicate.

Fig. 2. Inhibitory effect of NNU-023, ACV, and DHPG on the production of HSV-2 (A) and HCMV (B).

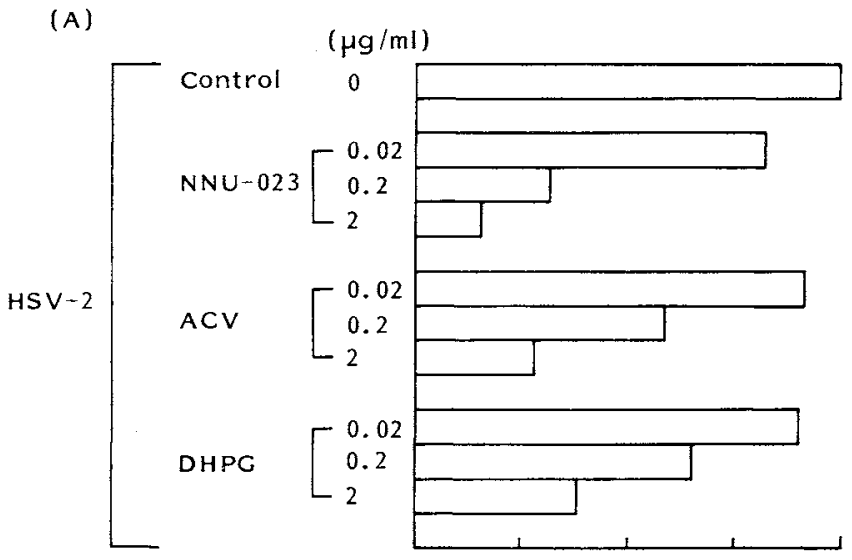

(B)

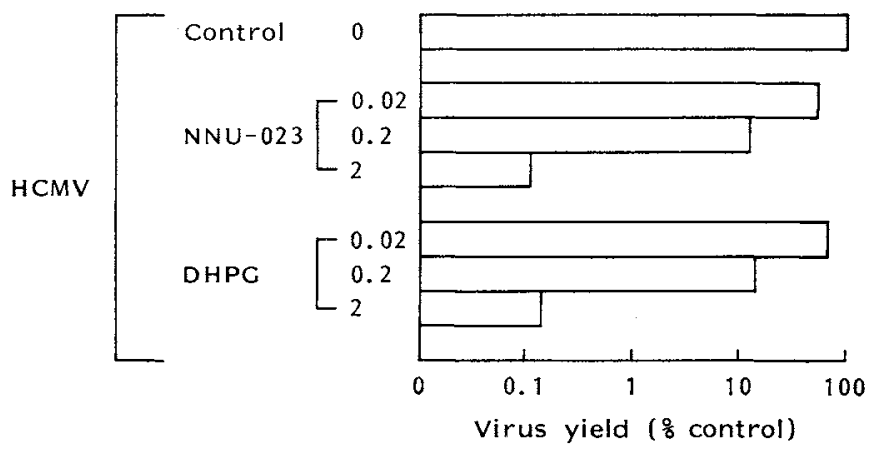

Vero or HEF cells were infected with either HSV-2 or HCMV at a multiplicity of infection of approximately $10 \mathrm{PFU} / \mathrm{cell}$, as described in Materials and Methods. The titers were the average of triplicate samples.

were expressed in terms of median effective concentrations $\left(\mathrm{EC}_{50}\right)$ which were defined as the drug concentrations that reduced viral replication to $50 \%$. The results are shown in Table 1. Among six compounds tested, NNU-023, carbocyclic OXT-G, was found to have the most potent anti-herpetic activity; the $\mathrm{EC}_{50}$ of this compound against HSV-2 and $\mathrm{HCMV}$ were approximately 0.04 and $0.40 \mu \mathrm{g} / \mathrm{ml}$, respectively. 
The $\mathrm{EC}_{50}$ value of NNU-023 against $\mathrm{HSV}-2$ was significantly lower than those of ACV and DHPG, while the $\mathrm{EC}_{50}$ value of this compound against HCMV was comparable to that of $\mathrm{DHPG}^{18 \sim 20)}$. Similar results were obtained in yield reduction assays where cells were infected with HSV-2 and HCMV at a relatively high multiplicity (10 PFU/cell) (Fig. 2), namely NNU-023 was much more active against HSV-2 than ACV and DHPG.

Effect of Carbocyclic OXT-G against Thymidine

Kinase Deficient ( $\mathrm{TK}^{-}$) Mutant of HSV-2

We compared the activity of NNU-023 with those of OXT-G, ACV and DHPG against wild-type and $\mathrm{TK}^{-}$HSV-2. As reported previously ${ }^{12)}$, OXT-G exhibited equal potencies against $\mathrm{TK}^{+}$and $\mathrm{TK}^{-}$ HSV-2, while the antiviral activity of $\mathrm{ACV}$ and DHPG was strikingly weakened by the lack of HSV-induced TK. Although carbocyclic OXT-G was still most active among four compounds against $\mathrm{TK}^{-} \mathrm{HSV}-2$, the $\mathrm{EC}_{50}$ value increased about 35 -fold compared with that against $\mathrm{TK}^{+} \mathrm{HSV}-2$ (Table 2).

\section{Reversal of Anti-herpetic Activity of NNU-023 by Exogenous Nucleosides}

The effect of the addition of exogenous nucleosides on the antiviral activity of NNU-023 was investigated to characterize the mode of action of this compound. Confluent monolayers of Vero cells were infected with about 100 PFU of HSV-2, and after a 1-hour virus adsorption period, cells were overlaid with $0.5 \%$ agarose containing NNU-023 and deoxyribonucleosides. As shown in Table 3, the antiviral activity of NNU-023 was partially reversed by the addition of 100 -fold excess deoxyguanosine (dG). The addition of four deoxynucleosides $(4 \mathrm{dN})$ all together was much more effective in reversing the antiviral effect of NNU-023 than that of $\mathrm{dG}$ alone. However, even 100-fold excess of $4 \mathrm{dN}$ did not induce complete reversion of antiviral effect of carbocyclic OXT-G.

\section{Discussion}

NNU-023 (carbocyclic OXT-G) was most potent against HSV-2 and HCMV among carbocyclic oxetanocins tested; the $\mathrm{EC}_{50}$ of $\mathrm{HSV}-2$ and $\mathrm{HCMV}$ was 0.04 and $0.40 \mu \mathrm{g} / \mathrm{ml}$, respectively. The activity of this compound against HSV-2 was significantly more potent than those of $\mathrm{ACV}$ and DHPG. However, the inhibitory concentration of the agent for the growth of Vero cells was lower than those of $\mathrm{ACV}$ and DHPG; NNU-023 inhibited the growth of Vero cells by $50 \%$ at the concentration of about $5 \mu \mathrm{g} / \mathrm{ml}$ (data not shown) and then the selectivity index was 125 . While OXT-G exhibited equal potencies against $\mathrm{TK}^{+}$ and $\mathrm{TK}^{-} \mathrm{HSV}-2$, carbocyclic OXT-G was about 35 -fold more active against $\mathrm{TK}^{+} \mathrm{HSV}-2$ than a $\mathrm{TK}^{-}$ mutant. This observation suggests that carbocyclic OXT-G, unlike OXT-G, is a good substrate for HSV-2-induced TK. Since carbocyclic OXT-G still showed the high activity against $\mathrm{TK}^{-} \mathrm{HSV}-2$, this nucleoside analog must be well phosphorylated by a nucleoside kinase of Vero cells too. Antiviral activity of NNU-023 was only partially reversed even with 100 -fold excess of $\mathrm{dG}$, and the addition of $4 \mathrm{dN}$ was shown to be much more effective in reversing antiviral activity than that of $\mathrm{dG}$ alone. 
These results suggest that the antiviral effect of carbocyclic OXT-G can not be solely due to its ability to act as a dG analog. On the other hand, the antiviral activity of OXT-G can be reversed more effectively by the addition of $\mathrm{dG}$ alone (unpublished observation). It appears that the mode of action of carbocyclic OXT-G is different from that of OXT-G. In addition to these observations, our preliminary in vivo studies have shown that the administration of NNU-023 at a dose of $5 \mathrm{mg} / \mathrm{kg} /$ day is highly effective in reducing the mortality rate of HSV-2-infected mice. When evaluated under the same conditions, on the other hand, ACV had little or no effect on the mortality rate even at the dose of $50 \mathrm{mg} / \mathrm{kg} / \mathrm{day}^{13}$. We also found that NNU-023 did not induce any overt signs of toxicity in mice, which received intraperitoneally the drug at a dose of $20 \mathrm{mg} / \mathrm{kg} /$ day for 10 days. We thus conclude that NNU-023 is a compound worth further investigation.

\section{Acknowledgment}

We thank K. MAENo for encouragement and T. Tsuruguchi and E. IWATA for technical assistance.

\section{References}

1) Schaeffer, H. J.; L. Beauchamp, P. De Miranda, G. B. Elion, D. J. Bauer \& P. Collins: 9-(2-Hydroxyethoxymethyl)guanine activity against viruses of the herpes group. Nature 272: 583 585, 1978

2) Elion, G. B.: Mechanism of action and selectivity of acyclovir. Am. J. Med. 73: 7 13, 1982

3) Crumpacker, C. S.; L. E. Schnipper, J. A. Zaia \& M. J. Levin: Growth inhibition by acycloguanosine of herpesviruses isolated from human infections. Antimicrob. Agents Chemother. 15: 642 645, 1979

4) Mar, E. C.; P. C. Patel \& E. S. Huang: Effect of 9-(2-hydroxyethoxymethyl)guanine on viral-specific polypeptide synthesis in human cytomegalovirus-infected cells. Am. J. Med. 73: 82 85, 1982

5) Collaborative DHPG Treatment Study Group: Treatment of serious cytomegalovirus infections with 9-(1,3-dihydroxy-2-propoxymethyl)guanine in patients with AIDS and other immunodeficiencies. N. Engl. J. Med. 314: $801 \sim 805,1986$

6) Laskin, O. L.; C. M. Stah-Bayliss, C. M. Kalman \& L. R. Rosecan: Use of ganciclovir to treat serious cytomegalovirus infections in patients with AIDS. J. Infect. Dis. 155: 323 327, 1987

7) KeAY, S.; J. BisseT \& T. C. Merigan: Ganciclovir treatment of cytomegalovirus infections in iatrogenically immunocompromised patients. J. Infect. Dis. 156: 1016 1021, 1987

8) Chachoua, A.; D. Dieterich, K. Krasinski, J. Greene, L. Laubenstein, J. Wernz, W. Buhles \& S. Koretz: 9-(1,3-Dihydroxy-2-propoxymethyl)guanine(ganciclovir) in the treatment of cytomegalovirus gastrointestinal disease with the acquired immunodeficiency syndrome. Ann. Intern. Med. 107: 133 137, 1987

9) Shepp, D. H.; P. S. Dandliker, P. De Miranda, T. C. Burnette, D. M. CederberG, L. E. KirkK \& J. D. Meyers: Activity of 9-\{[2-hydroxy-1-(hydroxymethyl)ethoxy]methyl $\}$ guanine in the treatment of cytomegalovirus pneumonia. Ann. Intern. Med. 103: $368 \sim 373,1985$

10) REeD, E. C.; P. S. DAudliker \& J. D. Meyers: Treatment of cytomegalovirus pneumonia with 9-\{[2-hydroxy-1-(hydroxymethyl)-ethoxy]methyl $\}$ guanine and high-dose corticosteroids. Ann. Intern. Med. 105: $214 \sim 215,1986$

11) ReED, E. C.; D. H. Shepp, P. S. DAndliker \& J. D. Meyers: Treatment of cytomegalovirus (CMV) infection of the gastrointestinal (GI) tract with 9-[2-hydroxy-1-(hydroxymethyl)ethoxymethyl]guanine (BW 759U). Program and Abstracts of the 26th Intersci. Conf. on Antimicrob. Agents Chemother., No. 567, p. 200, New Orleans, Sept. $28 \sim$ Oct. 1,1986

12) Nishiyama, Y; N. Yamamoto, K. Takahashi \& N. Simada: Selective inhibition of human cytomegalovirus replication by a novel nucleoside, oxetanocin $\mathrm{G}$. Antimicrob. Agents. Chemother. 32: 1053 1056, 1988

13) Nishiyama, Y.; N. Yamamoto, Y. Yamada, H. Fujoka, N. Shimada \& K. TaKahashi: Efficacy of oxetanocin G against herpes simplex virus type 2 and murine cytomegalovirus infections in mice. J. Antibiotics 42: 1308 1311, 1989

14) Shimada, N.; S. Hasegawa, S. Saito, T. Nishikiori, A. Fuj \& T. Takita: Derivatives of oxetanocin: Oxetanocins $H, X$ and $G$, and 2-aminooxetanocin A. J. Antibiotics 40: 1788 1790, 1987

15) Ichikawa, Y.-I; A. Narita, A. Shiozawa, Y. Hayashi \& K. Narasaka: Enantio- and diastereoselective synthesis of carbocyclic oxetanosin analogues. J. Chem. Soc. Chem. Commun. 1989: 1989, in press.

16) Nishiyama, Y.; K. MaENo \& S. Yoshida: Characterization of human cytomegalovirus-induced DNA polymerase and the associated 3'-to-5'-exonuclease. Virology 124: $221 \sim 231,1983$

17) Nishiyama, Y.; S. Suzuki, M. Yamauchi, K. Maeno \& S. Yoshida: Characterization of an aphidicolin-resistant mutant of herpes simplex virus type 2 which induces an altered viral DNA polymerase. Virology 135: 87 96, 1984 
18) MaR, E.-C.; Y.-C. ChenG \& E.-S. Huang: Effect of 9-(1,3-dihydroxy-2-propoxymethyl)guanine on human cytomegalovirus replication in vitro. Antimicrob. Agents Chemother. 24: 518 521, 1983

19) Field, A. K.; M. E. Davies, C. Dewitt, H. C. Perry, R. Lion, J. Germershausen, J. D. Karkas, W. T. Ashton, D. B. R. JoHUSTON \& R. L. Tolman: 9-(\{2-Hydroxy-1-(hydroxymethyl)ethoxy]methyl $\}$ guanine: a selective inhibitor of herpes group virus replication. Proc. Natl. Acad. Sci. U.S.A. 80: 4139 4143, 1983

20) Smee, D. F.; J. C. Martin, J. P. H. Verheyden \& T. R. Matthews: Anti-herpesvirus activity of the acyclic nucleoside 9-(1,3-dihydroxy-2-propoxymethyl)guanine. Antimicrob. Agents Chemother. 23: 676 682, 1983 